\title{
La crisis de la Unión Europea y el auge de la extrema derecha
}

\author{
Gonzalo Rodríguez Martínez \\ UNIVERSIDAD DE SANTIAGO DE COMPOSTELA, GALICIA, ESPAÑA
}

Resumen: La Unión Europea se encuentra inmersa en un proceso de reestructuración profundo debido a la irrupción de nuevas formaciones políticas de extrema derecha tanto en el plano estatal como en el comunitario, y al contrario que en anteriores ocasiones este proceso viene a debilitar más que a fortalecer todo lo que implica el proyecto de integración europeo. Ante el gran desafío que implica esta oleada de euroescepticismo, el presente trabajo viene a plantear la necesidad de creación de nuevas formas de legitimidad para el proyecto e instituciones europeas a través de la reformulación del concepto de ciudadanía europea y de la recuperación de los valores sociales y democráticos fundacionales del proyecto.

Palabras clave: Unión Europea, ciudadanía, legitimidad, crisis, extrema derecha.

Abstract: The European Union is immersed in a process of deep restructuring due to the emergence of new political right-wing formations both at the state and community level, and unlike in previous occasions this process comes to weaken rather than strengthen everything which implies the European integration project. In view of the great challenge that this wave of Euroscepticism implies, this work raises the need to create new forms of legitimacy for the European project and institutions through the reform of the concept of European citizenship and the recovery of social and democratic values founders of the project.

Keywords: European Union, citizenship, legitimacy, crisis, far-rights parties

\section{Introducción}

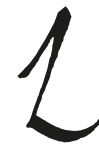

a Unión Europea es el proyecto más ambicioso en términos políticos y económicos de la historia reciente del mundo occidental. Tras dos guerras mundiales, la muerte de millones de personas y el reto de reconstruír un continente en ruinas, Europa se enfrenta al desafío de generar un periodo prolongado de paz a través de un proceso de integración de mercados y economías que sustituyesen los procesos competitivos por dinámicas de cooperación entre los países europeos. Ese reto termina desembocando en un proyecto político cuya vocación es trascender fronteras y hacer menguar los sentimientos nacionalistas excluyentes que tanto daño habían causado en el pasado reciente. Como toda gran idea, la Unión Europea 
tiene nombres y apellidos de hombres y mujeres capaces de imaginar un futuro mejor para el conjunto de Europa, inicialmente cabría destacar a líderes como Jean Monnet, Konrad Adenauer o Robert Schuman, mientras que con el paso de los años, fueron Jacques Delors o Felipe González quienes recojen el testigo de liderar la construcción de la Unión Europea tal y como la conocemos hoy en día.

Los tratados constitutivos de Roma, firmados en 1957 y ratificados en el 1958, conforman junto al Tratado de París que establece la Comunidad Económica del Carbón y el Acero (CECA) y la Comunidad Europea de la energía atómica (EURATOM) los pilares fundamentales de la Unión Europea. Otros de los grandes hitos en forma de tratado de la Unión Europea son los Tratados de Maastritch o Lisboa en donde la UE adopta la moneda única y asume personalidad jurídica propia como institución comunitaria para actuar de forma eficiente e independiente en las relaciones internacionales. El proyecto de integración europeo no se topa con grandes problemas a la hora de construir la Unión Europea que conocemos hoy en día, no obstante, y obviando el precedente del fracaso a la hora de intentar rubricar una Constitución Europea en común para toda la unión, podría decirse que hasta el estallido de la crisis económica de 2008 y las consecuencias derivadas de la gestión de la misma, así como de otras crisis como la que se refiere a las actuales dinámicas de migración, la Unión Europea no había mostrado sus debilidades, que como se demostraría a continuación son de carácter estructural y suponen una amenaza para el funcionamiento y para la futura existencia del proyecto.

El paper que se presenta a continuación tiene como objetivo determinar cuales son estas disfunciones estructuralmente problemáticas y preguntarse acerca de las posibles alternativas o soluciones para que la actual situación de resurgimiento de fuerzas antieuropeístas de extrema derecha no termine por destruír un proyecto fundado sobre las ideas y valores del Estado social y democrático de derecho.

\section{El auge de la extrema derecha}

\subsection{Hacia una definición operativa}

Son muchos los trabajos que han abordado el estudio de los partidos de extrema derecha o derecha radical a lo largo de los ultimos años, y por lo tanto, existen diferentes interpretaciones ya no acerca del funcionamiento o naturaleza de dichos partidos políticos, sino con lo que respecta a su propia definición. Después de una revisión bibliográfica al respecto en el presente trabajo, designaremos a los partidos objeto de nuestro estudio como de extrema derecha y no como derecha radical, derecha radical populista u otras propuestas realizadas por diversos autores como Betz (2004) o Mudde (2007) al buscar generar un nexo entre los actuales partidos y aquellos que operaban en el periodo de entreguerras (Hainsworth, 1992). Dichos partidos, para 
ser definidos como de extrema derecha deben cumplir, al menos, con dos requisitos fundamentales según Piero Ignazi (1993), en primer lugar deben ser observados y ubicados por la ciudadanía y la academia como partidos de extrema derecha dentro de una escala de ubicación ideológica y en segundo lugar, sus posiciones ideológicas deben ser extremas en tanto que claramente diferenciables de sus competidores dentro del sistema de partidos. Algunas de sus características básicas serán el rechazo a cualquier forma o visión política de izquierdas, ya sea de perfil socialista o comunista, la existencia de un liderazgo fuerte dentro del partido (Acha, 1997) y la constante alusión a un nacionalismo excluyente y binario que genera una idea de nosotros y ellos en confrontación (González-Páramo, 2018).

De otra parte, estos partidos de extrema derecha serán estudiados en su condición de europeos, es decir, en referencia a su postura con respecto a la existencia y funcionamiento de la Unión Europea y sus instituciones ${ }^{1}$. La actitud generalizada de los partidos de extrema derecha hacia el proyecto europeo es de oposición ya que en su ideología nacionalista niega cualquier forma de cesión soberana hacia una entidad supranacional como la Unión Europea, y si bien dicha oposición puede ser más fuerte o más débil, este componente debe cumplirse para poder hablar de partidos euroescépticos (López, 2018), concepto que ha sido definido por Szcerbiak y Taggart como el conjunto de actitudes políticas contrarias al proceso de integración europeo (2008) y que abordaremos con mayor detalle en el siguiente apartado.

En último lugar, catalogaremos también a estos partidos de extrema derecha como nuevos, entendiendo como nuevo partido aquel que genera una modificación sustancial de un sistema de partidos determinado al introducir no solo un nuevo competidor electoral sino nuevos issues de debate (Harmel, 1985) tales como el rechazo a la globalización, la multiculturalidad o la reivindicación de una identidad nacional excluyente (Acha, 1997)

\subsection{Del consenso permisivo al euroescepticismo}

Los inicios de la Unión Europea se sustentan sobre lo que Lindber y Scheingold (1970) acuñaron como permissive consensus o consenso permisivo, concepto que venía a plantear, por un lado la existencia de consenso entre las diferentes elites políticas europeas al margen de su posicionamiento ideológico ${ }^{2}$ con respecto a la necesi-

1. Si bien este trabajo contextualiza los partidos de extrema derecha en su condición de europeos, existe numerosa literatura que ha sido consultada para la elaboración de este paper que aborda este fenómeno desde perspectivas nacionales, ideológicas u otras cuestiones significativas.

2. A excepción de los partidos comunistas y nacionalistas que eran contrarios a cualquier tipo de cesión de soberanía, ya fuese por una cuestión identitaria o bien porque dicha cesión supondría una reducción de la capacidad de presión por parte de la clase trabajadora sobre las burguesías nacionales. 
dad de construir un actor europeo capaz de competir directamente con las grandes super potencias y defender los intereses comunes de los diferentes países miembro, y permisivo en tanto que la confianza ciudadana en los protagonistas del proceso de integración era muy elevado en aquellos momentos por los que la legitimidad emanaba de su propia autoridad y apenas era necesario constuír fórmulas legitimadoras alternativas.

Evidentemente la idea de consenso o de aceptación de las ideas mayoritarias con lo que respecta a una cuestión determinada, también supone la existencia de minorías contrarias a dicho consenso, y en el caso de la Unión europea no iba a ser diferente. Casi desde el origen de la construcción de la Unión Europea podemos encontrar ejemplos de disconformidad con los diferentes avances hacia un continente más cohesionado política y económicamente hablando, pero no es hasta 1992 cuando dicha disconformidad se hace patente a la hora de ratificar el Tratado de Maastritch que es votado en referendum por los ciudadanos de los diferentes países miembro. Los resultados son mayoritariamente positivos, no obstante, en Francia, uno de los países impulsores de la Unión Europea, el si se impuso al rechazo del tratado por apenas medio millón de votos y en Dinamarca, aunque también de manera muy apretada, se rechaza la ratificación del tratado en primera instancia, para ser aprobado un año después por una mayoría suficiente.

A lo largo de los años, estas minorías han ido construyendo un espacio de oposición al movimiento europeísta, y con el estallido de la crisis económica de 2008 y sus posteriores efectos sobre las políticas europeas, un fenómeno que se daba principalmente en partidos de carácter nacionalista o comunista se convierte en una actitud palpable dentro de facciones de los grandes partidos tradicionales de los diferentes sistemas políticos europeos (Álvarez, 2012). A través de la consolidación de un discurso crítico en torno a la gestión de la crisis económica por parte de las instituciones comunitarias comienza un proceso de devaluación del nivel de confianza de los ciudadanos europeos sobre la Unión Europea como así se muestra en el gráfico 1. Como se puede observar, los niveles de confianza previos al estallido de la crisis se encontraban en máximos históricos, llegando a alcanzar en 2007 tasas del 57\% de confianza, con el estallido de la crisis en 2008 comienza el desmoronamiento de dicha confianza, que alcanza sus cotas más bajas durante el proceso de gestión de la crisis de deuda soberana en Grecia entre 2012 y 2013 donde la desconfianza llega a elevarse hasta el $60 \%$ de la ciudadanía. En la actualidad, los niveles de confianza han tendido a equilibrarse debido al enfriamiento de la crisis económica, no obstante, aquel consenso permisivo ha tornado en un euroescepticismo generalizado y que debe ser abordado por la Unión Europea. 


\section{Gráfico 1}

Evolución confianza en la UE 2005-2019

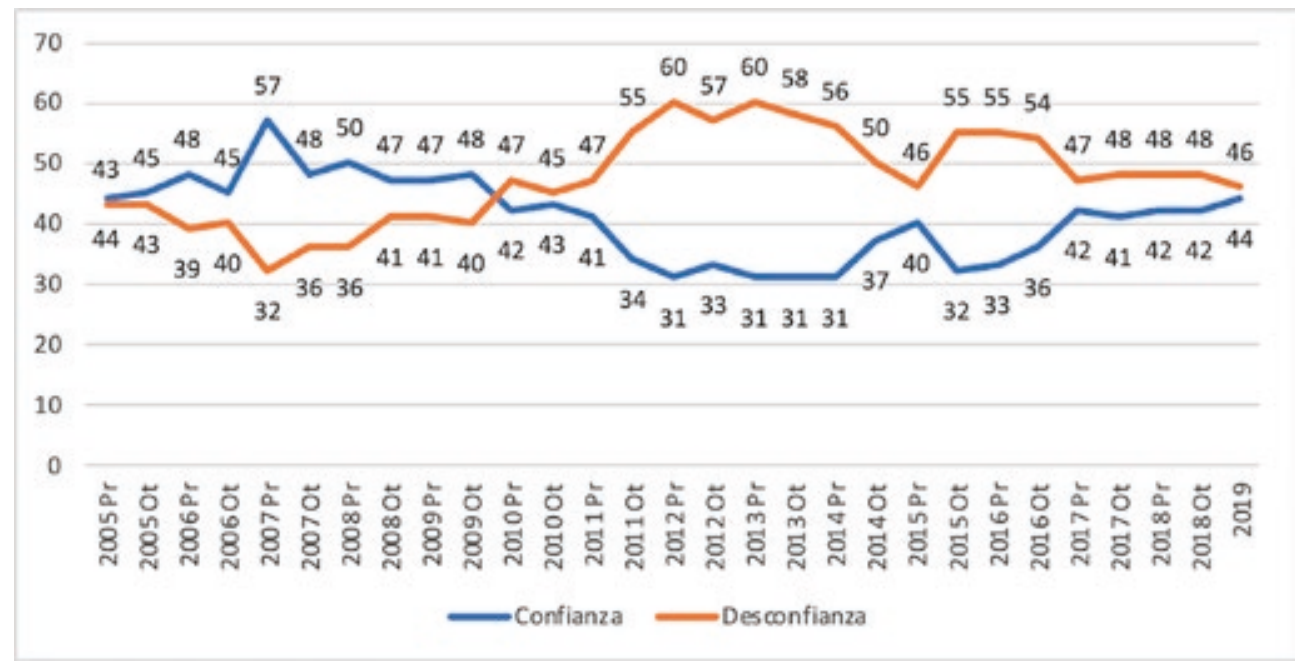

Fuente: Elaboración propia a partir de Eurobarómetro (63-90)

Asi por tanto, lo que anteriormente eran minorías discordantes han sido capaces de generar mayorías suficientes para competir con los grandes partidos tradicionales de los diferentes sistemas de partidos estatales, veáse el ejemplo de Francia con el Front National o el caso español con VOX y su reciente éxito electoral al convertirse en la tercera fuerza más votada en las elecciones del pasado 2019. En todo caso, si tuviesemos que destacar un movimiento euroesceptico capaz de generar mayorías transversales a todos los niveles políticos y sociales, debemos hacer alusión al Brexit, o lo que es lo mismo, a los resultados del referendum del 23 de Junio de 2016 en el que un $51,9 \%$ de los británicos votaron si a abandonar la Unión Europea, mientras que un $48,1 \%$ votaron para permanecer dentro de la comunidad europea. Al margen de los niveles de participación (72\%) y del escaso margen de la victoria rupturista, en la actualidad se está negociando la salida de Gran Bretaña de la Unión Europea y no parece existir la posibilidad de replantearse dicha decisión. El Brexit no es únicamente un fenómeno político o económico que hace referencia al descontento de un sector determinado de la población británica, sino que sus efectos tienen influjo sobre el resto de sistemas políticos europeos en forma de expansión de ideas nacionalistas desconsideradas con los principios de solidaridad que construyen las diferentes sociedades europeas tanto en su dimensión estatal como un su participación en la Unión Europea (Martino, 2016) 


\subsection{Elementos explicativos del auge de la extrema derecha}

Los resultados electorales dados en los diferentes países de la Unión Europea durante los últimos años vienen a apoyar la evidencia repecto al auge y consolidación de numerosos proyectos políticos de extrema derecha contrarios a la Unión Europea, tanto es así que en la actualidad únicamente tres de los países miembro de la unión no cuentan con un partido de extrema derecha en sus cámaras de representantes, a saber, Portugal, Irlanda y Luxemburgo. Por el contrario, son muchos los estados en los que la extrema derecha forma parte, en coalición o como apoyo, de los gobiernos nacionales, véase el ejemplo de Hungría, Polonia o Italia como parte de gobierno, o los casos de Francia y Alemania, dos de los principales protagonistas del proyecto europeo en donde la extrema derecha es la principal fuerza de la oposición y ha obligado en el caso alemán a lo que se conoce como gran coalición, es decir, a un pacto entre los dos principales partidos de la historia reciente alemana e ideológicamente enfrentados a gobernar juntos con la finalidad de evitar la entrada al gobierno de un partido de extrema derecha.

\section{Gráfico 2}

Papel de los partidos de extrema derecha en los sistemas políticos europeos
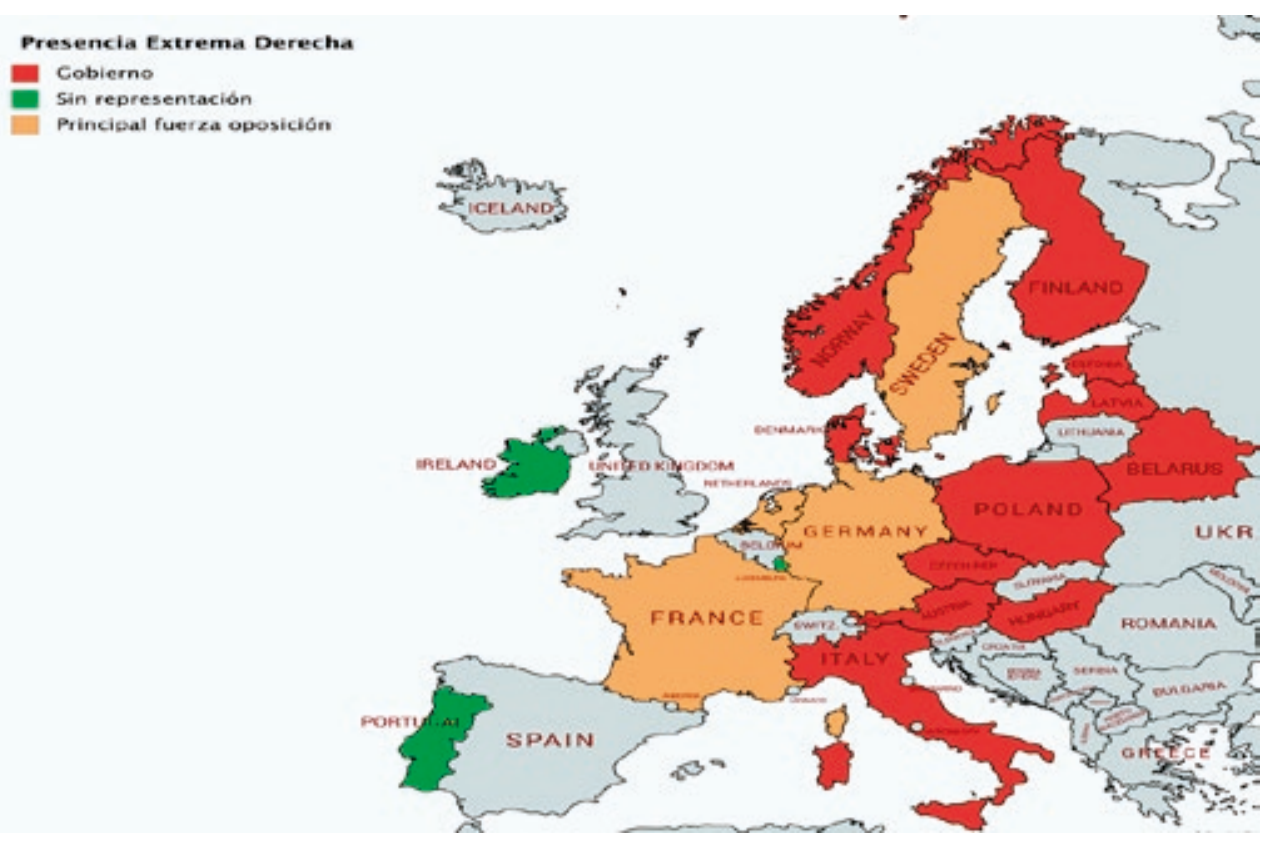
Cabría preguntarse entonces qué ha pasado, cómo es posible que el proyecto europeo que se ha basado históricamente en los derechos sociales y democráticos haya permitido el avance de estas fuerzas contrarias a todos los principios fundacionales de la Unión Europea. Como en todo fenómeno social, las causas son múltiples, no obstante, a continuación se plantearán algunas de las cuestiones fundamentales que nos permitirán aportar algo de luz y llegar a comprender este fenómeno de estudio.

La revolución derivada del asentamiento del postmaterialismo en las sociedades occidentales trajo consigo la aparición de partidos políticos para defender esas nuevas sensibilidades (Inglehart, 1991), como por ejemplo, los partidos verdes que vienen a defender las ideas del ecologismo o los partidos de perfil animalista que centran su actividad política en la defrensa de los derechos de los animales. Al mismo tiempo, se va construyendo un movimiento de oposición que nace para confrontar estas nuevas sensibilidades (Ignazi,1992) y precisamente de esta oposición es de donde nacen los nuevos partidos de extrema derecha.

De la creación de estos nuevos partidos, que defienden nuevas identidades o sensibilidades políticas se deriva tambien la creación de nuevos issues o temas de debate que deben ser abordados por los partidos políticos. Con lo que respecta al estudio de los nuevos partidos de extrema derecha en el marco de la Unión Europea tendríamos que referirnos a la gestión de fronteras y a los procesos de migración ya que estos temas son los que ha reconfigurado y polarizado a las diferentes sociedades europeas y han permitido expandir su mensaje a la extrema derecha de tal manera que un issue se transforma en cleavage mediante un proceso de politización premeditada de estos nuevos partidos (Hauss y Rayside, 1978). De una parte, se establece una visión abierta cuya vocación es progresista e inclusiva, es decir, que defiende las tesis del multiculturalismo, en cambio, en el lado opuesto nos encontramos con una visión cerrada, excluyente y nacionalista que trata de impedir las inmigraciones a lo que considera su propio territorio (González-Páramo, 2018). Esta última visión cerrada es la que asumen los nuevos partidos de extrema derecha que difunden informaciones que criminalizan y legitiman la exclusión de los colectivos migrantes con la finalidad de construír una sociedad cerrada de valores previos a la globalización que implicarían una supuesta mejora de la economía y por tanto de la calidad de vida de los "incluídos" ya que dentro de la retórica de la extrema derecha los migrantes son los principales causantes y beneficiarios de la crisis económica que padecen los ciudadanos medios.

A pesar de la heterogeneidad de los sistemas políticos europeos, los nuevos partidos de extrema derecha han sido capaces de integrarse con mayor o menor acierto en los sistemas de partidos tradicionales con planteamientos anti sistema que los han llevado de los márgenes al centro de todos los debates politicos. Resulta evidente que 
cada éxito de un partido contrario a todos los consensos democráticos y sociales propios de las sociedades europeas, implica una derrota para todos aquellos que se denominan demócratas. Como estudia Kriesi (1992) a través de la conceptualización de la estructura formal institucional del Estado son muchos los factores que pueden facilitar o debilitar la aparición de estos partidos pero sin duda, alguno de los más importante son los sistemas electorales, la capacidad de financiación, la reacción del sistema de partidos ante la aparición de estos nuevos competidores y la cobertura mediática proporcionada a dichos partidos.

En primer lugar, el sistema electoral determina en gran medida la capacidad de surgimiento de los partidos de extrema derecha, para estos un sistema proporcional es más favorable que uno mayoritario ya que la posibilidad de obtener representación aumenta cuanto más fragmentado y cuantos menos requisitos se exigan para el acceso a escaños (Acha, 1997), como muestra de ello podemos observar el caso francés, en el que la existencia de un sistema mayoritario con dos vueltas permite a Macron vencer a Marine Le Pen o a Chirac vencer a Jean Marie Le Pen al congregar todo el voto de las fuerzas demócratas moderadas en segunda vuelta para evitar el ascenso de un partido de corte extremista. De la capacidad de obtener representación se deriva en muchos casos la capacidad de financiarse, por lo que la existencia de una fiscalización de los fondos públicos destinados a movimientos y partidos políticos puede suponer un freno al surgimiento de partidos de contrarios a los principios fundamentales de cualquier sistema democrático.

Otro elemento fundamental para comprender la aparición de estos nuevos partidos de extrema derecha es la reacción de los otros actores políticos del sistema de partidos ante la aparición de partidos de extrema derecha y de sus nuevos issues. La academia no encuentra consenso al respecto de como reaccionar ante este nuevo fenómeno, pero en todo caso la discusión gira en torno a dos posturas, la que plantea que debatir con partidos extremos normaliza su situación e incrementa el electorado potencial de los mismos (Berrington, 1985) y la que plantea que no confrontarlos implica dejar que todo un espacio político electoral se radicalice (Acha, 1997).

Por último, los medios de comunicación dentro de sistemas políticos cada vez más mediatizados son un elemento diferencial a la hora de aumentar o disminuir la capacidad de influencia de un partido político de extrema derecha, así por tanto, la existencia de unos medios de comunicación consecuentes para con sus valores democráticos supondrá una dificultad añadida para los nuevos partidos de extrema derecha, mientras que la existencia de medios de comunicación de carácter más sensacionalista supondrán un elemento facilitador a la hora de expandir ciertos discursos. 


\subsection{Elementos ideológicos de los nuevos partidos de extrema derecha}

Los nuevos partidos de extrema derecha guardan similitudes y simpatías con los viejos partidos de de extrema derecha del periodo de entreguerras, no obstante, su estrategia formal sufre una transformación al verse reducida su capacidad de influencia a grupúsculos marginales minoritarios política y socialmente hablando. Cas Mudde (2007) plantea que la nueva extrema derecha es nominalmente democrática, pero esencialmente contraria a la democracia liberal y es desde este giro estratégico desde el que esta nueva familia de partidos desplega su ideología y discurso.

Los elementos ideológicos centrales de esta nueva familia de partidos de extrema derecha siguen siendo el nacionalismo y el autoritarismo, no obstante, estas dos toman nuevas formas para así ser capaces de no generar rechazo en las nuevas poblaciones post industriales de valores democráticos asentados.

En primer lugar, el nacionalismo torna en lo que Betz (2004) denomina como nativismo, "una ideología que viene a plantear que los estados deberían ser habitados exclusivamente por miembros del grupo nativo y que los elementos no - nativos (personas e ideas) son una amenaza para un estado nación homogéneo" (Mudde, 2007:19). Por tanto, ya no se hacen alusiones directas a la cuestión racial o nacional pero el discurso continúa siendo esencialmente xenófobo aunque se oculte tras una cortina de eufemismos en alusión a la legalidad o a las necesidades primarias de las economías nacionales. Veánse por ejemplo las medidas propuestas por el partido político VOX en materia de inmigración contenidas en su programa electoral para las ultimas elecciones parlamentarias en España:

- Deportación de inmigrantes ilegales a sus países de origen.

- Deportación de inmigrantes legales que hayan reincidido en la comisión de delitos leves o hayan cometido algún delito grave.

- (...)Cualquier inmigrante que haya entrado ilegalmente a España estará incapacitado de por vida a legalizar su situación y a recibir cualquier tipo de ayuda de la administración.

- La inmigración se afrontará atendiendo a las necesidades de la economía espanola y a la capacidad de integración del inmigrante.

En segundo lugar otra característica básica de la extrema derecha es el autoritarismo, elemento contrario a todos los ideales democráticos construidos tras la derrota del fascismo en la Segunda Guerra Mundial. Ahora el perfil autoritario debe transformarse para ser admitido por los sistemas liberales europeos (Antón-Mellón, 2007). En este sentido apunta Roger Griffin (2000), quien denomina este fenómeno como liberalismo etnocrático que viene a plantear la "toxicidad" de estos nuevos partidos para con los sistemas de partidos democráticos en al menos dos cuestiones fundamentales. Con respecto a su posición de competidor electoral, lo que posibilita un 
gobierno de extrema derecha como el que ya existe en Polonia y Hungría y que suponen una amenaza para la democracia, y con respecto también, a la acción de contagio de la agenda política de los partidos de extrema derecha sobre el resto del sistema de partidos. Recurriendo de nuevo al caso español, es sorprendente como VOX es capaz de marcar la agenda del Partido Popular o de Cidadanos con sus temas de debate en referencia a la violencia machista o al adoctrinamiento en las escuelas públicas españolas.

\section{Unión Europea: crisis de legitimidad y fórmulas para recuperarla}

Hasta el momento nos hemos preguntado acerca del surgimiento de los nuevos partidos de extrema derecha. A continuación trataremos de plantear la ventana de oportunidad abierta por la Unión Europea como institución a la hora de facilitar la aparición y consolidación de estos partidos contrarios a los principios de Europa.

La Unión Europea nace debido a dos pretensiones claramente defensivas, por una parte con respecto a sus propios integrantes y con la finalidad de evitar una nueva guerra a escala regional, y en segundo término con respecto a la Unión Soviética y el miedo a una posible expansión generalizada del comunismo a lo largo y ancho del continente europeo. Y si bien el primero de los miedos parecía disiparse con el paso de los años, el conflicto con la Unión Soviética se enquista de tal manera que la Guerra Fría dura más de cuarenta años. Con el paso de los años el proyecto europeo toma impulso y en lugar de defenderse, adopta posiciones más ambiciosas de las que se derivan las cuatro libertades de circulación, a saber, de capitales, mercancías, servicios y de personas. En último lugar, la puesta en marcha del euro termina de construír un mercado común, con una moneda única y gestionada desde un Banco Central Europeo.

El proyecto parecía avanzar de manera firme, no obstante, resulta evidente que mientras la pertenencia a la Unión Europea solo implicaba ventajas, no existiría ningún movimiento anti europeo, pero con la llegada de la crisis en 2008 se pondrían de manifiesto al menos tres grandes problemas de carácter estructural que vienen a poner en riesgo el futuro de la Unión Europea (Castells, 2017).

En primer lugar, la falta de una identidad europea en común(Innerarity, 2014), no existe un demos europeo, existe un territorio y existen decenas de comunidades a las que no se ha podido o no se ha sabido cohesionar ni en términos identitarios ni culturales. En segundo lugar, todo el proceso de integración se construye al margen de la ciudadanía europea en lo que Castells (2017) llega a denominar como una forma de despotismo ilustrado moderno, que fue posible gracias al consenso permisivo que ya abordamos con anterioridad. Y por último, el euro, una moneda 
única para economías brutalmente heterogéneas ${ }^{3}$ incapaces de asimilar las políticas impuestas desde la Unión Europea y crecer de manera sostenible. Tanto es así que la política monetaria y las medidas de austeridad propuestas como salida de la crisis económica terminan por quebrar la opinión pública de la Unión Europea con respecto al futuro de Europa (Arrizabalo, 2016), incluso algunos autores como Joseph Stiglitz (2017) llegan a denominar el euro como un elemento autodestructivo para la Unión Europea.

En este marco de fragmentación cultural/identitaria donde los ciudadanos no se sienten parte del proceso democrático y en donde la crisis económica ha demostrado todos los errores cometidos en el proceso de integración económica surgen los nuevos partidos de extrema derecha que solo necesitan de un issue favorable para desplegar su discurso. Retomando la idea del cleavege abierto - cerrado anteriormente planteado podemos deducir como ante las lagunas estructurales de la Unión Europea y una crisis humanitaria como la que se está viviendo en torno a los refugiados, los partidos de extrema derecha tienen un terreno favorable para movilizar electorado descontento con la gestión de la crisis y al que se le induce a pensar que europa y los inmigrantes son los principales responsables de todos los posibles problemas padecidos por la ciudadanía europea.

Para generar mayores tasas de legitimidad han de abordarse los conflictos que generan desafección para con el proyecto de integración europeo, y como decíamos anteriormente, uno de los principales factores a la hora de explicar el auge de los nuevos partidos de extrema derecha en Europa era la gestión de la crisis económica de 2008, así por tanto, se deben generar mecanismos que intenten evitar una nueva crisis y sus efectos sobre la ciudadanía. Europa se encuentra en una encruzijada en la que debe decidir entre avanzar hacia una economía más integrada, o retroceder y disolver la eurozona tal y como se configura en la actualidad. Asumiendo la complejidad y las implicaciones que poseen ambas opciones, en el presente trabajo se opta por una profundización en la integración europea cuya finalidad será generar un espacio más homógeneo y por tanto más abordable económicamente hablando. Algunas de las cuestiones más apuntadas por la literatura reciente a este respecto han sido:

- Unión Bancaria Europea (Stiglitz, 2017): La Unión Europea debe generar un sistema financiero comunitario sólido y en clave solidaria, que vele por los intereses de la UE en su conjunto para evitar los desequilibrios que en la actualidad ponen en riesgo las economías que más han sufrido la crisis. Es necesario un banco común que funcione como garrantía de los depósitos bancarios de

3. Cuestión que se agravará al no cumplirse los criterios de adhesión al euro impuestos por la propia Unión Europea. 
todos lo países miembro con la finalidad de evitar fugas de capitales desde los países periféricos a los países con economías más sólidas. Pongamos el ejemplo de un inversor o un ahorrador solvente que tiene su capital afincado en un país como España o Grecia y que debido a la libertad de circulación para los capitales decide comenzar a trabajar con bancos alemanes u holandeses para así garantizar su dinero, hablamos de una práctica habitual, pero que pone en riesgo las economías de los países que sufren esta fugas de capitales. Exactamente por esto, se hace tan importante que existe una entidad europea que garantice dichos depósitos. De otra parte, también sería interesante la unión bancaria a la hora de evitar contracciones o impulsos al crédito de manera errónea. Cuando una economía entra en crisis, sus actuales reguladores, es decir, los bancos privados restringen el crédito cuando los saludable para la economía en general serían políticas más expansivas. Por el contrario, cuando la economía crece, los bancos privados, ofrecen crédito más asequible por lo que estas economías entran en riesgo de procesos inflacionarios excesivos. En cambio si el regulador del crédito a nivel comunitario fuese una entidad de carácter público, se podría facilitar el crédito en momentos de crisis para evitar impagos y se podría restringir el crédito en momentos de expansión para fomentar el ahorro y evitar una inflación excesiva.

- Condonación y mutualización de deuda (Varoufakis, 2012): En primer lugar, debemos asumir que las economías del sur de Europa como Grecia o España cuyas obligaciones de deuda superan la totalidad de su PIB anual son evidentemente inasumibles e impagables si se pretende que los ciudadanos de dichos países sigan viviendo de acuerdo a los criterios del Estado de Bienestar. Así por tanto, Varoufakis plantea la necesidad de auditar las deudas de los diferentes países para determinar que parte de esas deudas es condonable solidariamente entre los diferentes países de la Unión Europea. Al mismo tiempo, se plantea la necesidad de que los bonos europeos con los que se financian los diferentes países europeos pasen a ser avalados de manera mutua, es decir, que el conjunto de la unión responda ante los endeudamientos de los diferentes países, para así, conseguir un crecimiento sostenible y razonable en toda la eurozona. En la actualidad, al acceso a financiación vía Europa es más barata para los países con economías saludables que para los países con necesidades económicas, lo cual, en clave de mercado es evidentemente correcto, pero en clave solidaria es una barbaridad que ahoga aún más a aquellos países que necesitan crecer.

- Un marco fiscal común: Es incongruente que cada Estado posea soberanía fiscal al mismo tiempo que la política monetaria es competencia europea, ya que esta realidad induce a una forma de competencia interna entre los diferentes 
países europeos. Por ejemplo, si una empresa multinacional, sea del sector que sea, decide trabajar o invertir en el marco de la Unión Europea nada le impide afincar su producción en un país como Portugal o Grecia donde los salarios son más bajos pero tener su sede fiscal en Irlanda donde paga menos impuestos por sus beneficios. Un marco fiscal común generaría igualdad de condiciones a la hora de atraer inversiones del extranjero y evitaría las actuales competiciones entre estados.

Igualmente importante resulta atajar los actuales conflictos de índole político que devaluan el carácter democrático de la Unión Europea para recuperar la confianza de la ciudadanía y poder retomar posiciones ambiciosas con lo que respecta a la profundización del proceso de integración europeo. Por ello, siguiendo las tesis de Habermas (2012) Europa debe avanzar hacia lo que el mismo denomina autodeterminación democrática, es decir, hacia una institucionalización efectiva de la toma de decisiones por parte de la ciudadanía.

Al igual que en la antiguedad se sustituyeron las identidades locales por las estatales a través de la creación de un demos, se vuelve ahora necesario generar un espacio que reduzca la distancia entre gobernantes y gobernados en el marco de la Unión Europea, donde un aumento de la deliberación del ciudadano genere un interés más elevado por la política europea y ello termine derivando en la cristalización de un ciudadanía europea como tal. A continuación se plantan algunas de las cuestiones más apuntadas desde la academia para reconstruír la legitimidad de las instituciones y de la Unión en su totalidad:

- Elecciones más democráticas y transparentes para los principales puestos de responsabilidad de la Unión Europea: Hix (2008) señala que esta medida generaría una mayor competencia entre las élites en disputa y que al mismo tiempo facilitaría una mayor implicación por parte de la ciudadanía europea al poner conocer las diferentes propuestas de cada uno de los candidatos a dichos puestos de responsabilidad.

- Aumento de la difusión mediática en los medios de comunicación: Según Follesdal y Hix (2006) el hecho de que los medios de comunicación de los diferentes países proporcionase una cobertura más exhaustiva de los procesos políticos acontecidos en la Unión Europea facilitaría que la ciudadanía mostrasde más interés al respecto de las cuestiones europeas. En la misma linea, también resulta interesante la creación de una televisión pública europea cuya vocación fuese fomentar el europeismo entre los ciudadanos de la unión.

- Javier Arregui (2012) propone tres reformas para las tres principales instituciones de la Unión Europea. En primer lugar, con respecto a la Comisión y su presidencia, Arregui considera que esta debería ser elegida directamente por 
la ciudadanía de tal manera que los candidatos tuviesen que presentar públicamente su perfil y sus propuestas ante todos los europeos. En referencia al Consejo, el autor considera que su principal disfunción es la opacidad con la que toma sus decisiones, y que estas deberían ser más transparentes para así generar confianza dentro de la ciudadanía. Por último, el Parlamento Europeo que es el órgano más netamente democrático de la Unión Europea debería convertirse en el órgano con mayor capacidad de decisión de la UE ya que es el único con la legitimidad suficiente para hacerlo.

\section{Conclusiones}

La crisis económica y las medidas propuestas por al Unión Europea para salir de la misma, unidas a la actual crisis migratoria han generado un clima de desconfianza dentro de la ciudadanía europea con las instituciones comunitarias. Esta cuestión, ha facilitado la aparición de nuevos partidos de extrema derecha que han utilizado este descontento de los ciudadanos para señalar culpables, migrantes e instituciones europeas, y plantear así soluciones simples antes situaciones enormemente complejas.

En el presente trabajo se han intentando señalar algunas de las cuestiones fundamentales tanto para comprender el auge de estos partidos, como para combatir sus ideas anti europeas y contrarias a las ideas sociales que fundamentan a la Unión Europea. Hemos comprobado que no existen fórmulas mágicas ni únicas, pero resulta evidente que una profundización de la cohesión europea en términos políticos, económicos y democráticos puede facilitar la vuelta a la normalidad democrática dentro de la Unión.

\section{Bibliografía}

ACHA, Beatriz (1997) "Partidos políticos de extrema derecha en Europa: Hacia un marco teórico para el análisis de los nuevos partidos", Estudios de Deusto, 45 (2) ANTÓN - MELLÓN, Joan y HERNÁNDEZ - CARR, Aitor (2016) "El crecimiento electoral de la derecha radical populista en Europa: parámetros ideológicos y motivaciones sociales", Política y Sociedad, 53, 17-28.

ARREGUI, Javier (2012) "Problemas de legitimidad democrática, representación y rendimiento de cuentas en el proceso político de la Unión Europea”, Cuadernos europeos de Deusto, 46.

ARRIZABALO, Xabier (2016) Capitalismo y economía mundial. Madrid: IME

CASTELLS, Manuel (2017) Ruptura. La crisis de la democracia liberal. Madrid: Alianza Editorial 
FORNER, Salvador y Heidy-Cristina SENANTE (2014) "La crisis de Europea y el ascenso del Populismo, Más allá de las elecciones europeas de 2014" Cuadernos de pensamiento político FAES, 43 (Julio)

GONZÁLEZ - PÁRAMO, Ana (2018) "El auge de la ultraderecha en Europea y los discursos xenófobos”, Fundación porCausa - Anuario CEIPAZ 2018-2019.

HABERMAS, Jürgen (2009). ¡Ay, Europa! Madrid: Editorial Trotta

HABERMAS, Jürgen (2012). La constitución de Europa. Madrid: Editorial Trotta

HAINSWORTH, Paul (1992). “The Extreme Right in Europe and the USA”. New York: St. Martin Press

HARMEL, Robert (1985). On the Study of New Parties. International Political Science Review, 6 (4).

HAUSS, Charles y David RAYSIDE (1978). "The Development of New Parties in Western Democracies since 1945: Development and Decay" Londres: Sage

HERNÁNDEZ - CARR, Aitor (2011). "La derecha radical populista en Europa: discurso, electorado y explicaciones" Reis, 136.

HILLEBRAND, Ernst (2016). "La crisis de la socialdemocracia europea", Nueva Sociedad, 261.

INNERARITY, Daniel (2014). Does Europe need a Demos to be truly democratic? LEQS Paper, 77.

INGLEHART, Ronald (1991). El cambio cultural en las sociedades industriales avanzadas. Madrid: CIS

KRIESI, Hanspeter, Ruud KOOPMANS, Jan WILLEM DUYVENDAK y Marco G. GIUGNI (1992). "New Social Movements and political opportunities in Western Europe". European Journal of Political Research, 22 (2).

LÓPEZ, Alberto (2017). "Brexit: La secesión de la Unión Europea entre teoría y realidad”. UNED: Teoría y Realidad Constitucional, 40: 111-140.

LÓPEZ, Santiago (2017). "Up your delors! El auge del euroescepticismo en Europa: ¿el fin de un proyecto o una crisis coyuntural?, Tiempo Presente. Revista de Historia, 5

MARTINO, Antonio (2016). “Brexit”. Revista POSTData (21)

PIERO, Ignazi (1995). The Extreme Right Parties in Western Europe. New York: Oxford University Press

SCZERBIAK, Aleks y Paul TAGGART (2008). "Opposing Europe? The Comparative Party Politics of Euroscepticism”. Oxford University Press, 6 (2)

STIGLITZ, Joseph (2017). El Euro. Cómo la moneda común amenanza el futuro de Europa. Barcelona: Penguin Random House. 
